Tropical Journal of Pharmaceutical Research December 2012; 11 (6): 939-945

(c) Pharmacotherapy Group, Faculty of Pharmacy, University of Benin

Benin City, 300001 Nigeria.

All rights reserved.

Available online at http://www.tjpr.org

Research Article

http://dx.doi.org/10.4314/tjpr.v11i6.10

\title{
Evidence for Enhanced Intestinal Absorption of Digoxin by P-Glycoprotein Inhibitors
}

\author{
Hadi Valizadeh ${ }^{1}$, Maryam Mehtari ${ }^{2}$ and Parvin Zakeri-Milani ${ }^{3 *}$ \\ ${ }^{1}$ Research Center for Pharmaceutical Nanotechnology, ${ }^{2}$ Drug Applied Research Center, ${ }^{3}$ Liver and Gastrointestinal \\ Diseases Research Center, Faculty of Pharmacy, Tabriz University of Medical Sciences, Tabriz, Iran.
}

\begin{abstract}
Purpose: To investigate the influence of macrolides as P-glycoprotein inhibitors on the level of intestinal absorption of digoxin.

Methods: Jejunal segments of anaesthetized rats were cannulated and perfused by digoxin in phosphate buffered saline (PBS) at $37^{\circ} \mathrm{C}$ in the presence or absence of macrolides (erythromycin and clarithromycin). Samples were obtained from outlet tubing at different time points and digoxin concentration assayed. The effective permeability of the drug was calculated after analyzing the samples using reverse-phase HPLC method.

Results: Digoxin effective permeability was in the range of $0.24 \pm 0.02 \times 10^{-4}$ to $0.32 \pm 0.06 \times 10^{-4}$ $\mathrm{cm} / \mathrm{sec}$ for the control group. The macrolides significantly $(p<0.05)$ increased intestinal transport of digoxin, with digoxin in the presence of $150 \mu \mathrm{M}$ of each macrolide in the range $0.42 \pm 0.08 \times 10^{-4}$ to 0.52 $\pm 0.07 \times 10^{-4} \mathrm{~cm} / \mathrm{sec}$. However, no significant difference $(p>0.05)$ was observed between the effects of the two macrolides.

Conclusion: The probable explanation for digoxin-macrolide interaction is inhibition of intestinal $P$ glycoprotein-mediated efflux of digoxin which leads to increased digoxin intestinal absorption.
\end{abstract}

Keywords: Digoxin, Macrolides, Efflux, Intestinal permeability, P-glycoprotein 


\section{INTRODUCTION}

P-glycoprotein (P-gp), a 170-kilodalton plasma membrane protein, belongs to the ATP-binding cassette superfamily. This protein acts as an energy dependent transmembrane drug efflux pump which is localized in a wide range of tissues including enterocytes of the gastrointestinal tract [1].

Studies in several species including rats have indicated that P-gp may play an important role in limiting drug absorption [2]. It limits the entry and/or accumulation of endogenous and exogenous P-gp substrates by active efflux. A large number of therapeutically and structurally disparate drugs are substrates for P-gp. Among these drugs digoxin has been well documented as a substrate for P-gp using in vitro and in vivo experiments [3, 4]. It is a cardiac glycoside which is widely used in the treatment of various heart conditions including atrial fibrillation, atrial flutter and congestive heart failure.

There is a variation in the absolute bioavailability of digoxin and this represents an important issue in drug development and therapy. Its intra and inter-individual bioavailability were reported to be in the range of $40-90 \%$ [5] and $50-85 \%$ [5], respectively. These variations have been associated with oral formulation factors such as particle size, drug polymorphism, and aging of products [5]. However, the mechanism of absorption of digoxin from intestinal membrane and the underlying cause of its highly variable bioavailability have not been fully addressed.

Macrolide antibiotics are used increasingly as first-line outpatient therapy for respiratory tract infections. Drug interactions involving these antibiotics include induction of hepatic metabolizing enzyme system and alteration of gastrointestinal flora [6]. There is evidence that certain macrolides, such as azithromycin and clarithromycin, are also inhibitors of apical drug efflux pump P-glycoprotein [7, 8]. However, the mechanism of macrolidedigoxin interaction is not fully understood.
Moreover, all available data for digoxinmacrolide interaction are from case reports which leads to increased risk of digoxininduced toxicity, including arrhythmias, anorexia, altered color vision, and mental changes.

To the best of our knowledge, there have been no controlled studies in vivo or in situ in this regard. Since macrolides used in reported cases were either erythromycin or clarithromycin, this study was aimed at assessing the effect of these macrolides on digoxin intestinal permeability. For this purpose, single-pass intestinal perfusion technique in anaesthetized rats was used. This approach is the most frequently used because it provides conditions closer to what to what obtains in humans following oral administration [9].

\section{EXPERIMENTAL}

\section{Chemicals}

Digoxin (Boehringer Ingelheim, Germany), as well as erythromycin and clarithromycin (Elder Pharmaceutical Ltd, India) were kindly provided by Zahravi Pharmaceutical Company. Acetonitril and methanol were of high performance liquid chromatography (HPLC) grade and obtained from Merck (Germany). Phenol red was purchased from Sigma Chemical Co (USA). $\mathrm{KH}_{2} \mathrm{PO}_{4}$, $\mathrm{NaH}_{2} \mathrm{PO}_{4}, \quad \mathrm{Na}_{2} \mathrm{HPO}_{4}, \mathrm{H}_{3} \mathrm{PO}_{4}, \mathrm{NaOH}, \mathrm{NaCl}$ were purchased from Merck (Germany). Deionized water was used for HPLC studies.

\section{Instrument}

A liquid chromatographic system (Beckman, USA) comprising of 126 gold solvent delivery module equipped with a Rheodyne (Cotati, California) injector and a variable wavelength ultraviolet 166 gold spectrophotometric detector (Beckman, USA) was used foe HPLC studies. The analytical column used for chromatographic separations was Shimpack VP-ODS $5 \mu \mathrm{m} 4.6 \times 250 \mathrm{~mm}$ (Shimadzu, Japan) with a Shimpack VP-ODS $5 \mu \mathrm{m} 4.6 \times$ 
$50 \mathrm{~mm}$ guard column (Shimadzu, Japan) for phenol red and $\mathrm{C}_{8}(4.6 \times 150)$ Lichrosphere reverse phase select $B$ column (Merck, Germany) for digoxin. The system's Gold software was used for data acquisition while Gold nouveau software was used for data reporting and analysis.

\section{Chromatographic conditions}

The mobile phase for digoxin was $26 \% \mathrm{v} / \mathrm{v}$ of acetonitrile in water which was filtered through sintered glass filter P5 (1.0 - $1.6 \mu$, Winteg, Germany) and degassed in a sonicator (Liarre, Italy) under vacuum. The mobile phase was pumped in isocratic mode at a flow rate of $2 \mathrm{ml} / \mathrm{min}$ at ambient temperature. UV detection was accomplished at $218 \mathrm{~nm}$ and samples of $100 \mu \mathrm{l}$ were injected using Hamilton injector syringe (Hamilton, Bonaduz, Switzerland) onto the column. For phenol red analysis, the mobile phase consisted of $45 \% \mathrm{v} / \mathrm{v}$ of $0.5 \mathrm{M} \mathrm{KH}_{2} \mathrm{PO}_{4}$ and $55 \% \mathrm{v} / \mathrm{v}$ methanol (adjusted to $\mathrm{pH} 2.6$ ) and the detector was set at $430 \mathrm{~nm}$ [9].

\section{Intestinal experiments}

In situ permeation studies were performed using established methods [9] Male Wistar rats $(200-250 \mathrm{~g})$ were maintained on $12 \mathrm{~h}$ light-dark cycle and fasted 12 - $18 \mathrm{~h}$ before each experiment although drinking water was readily accessible. They were anaesthetized using an intraperitoneal injection of sodium pentobarbital $(60 \mathrm{mg} / \mathrm{kg})$ and placed on a thermostatted heated pad to maintain normal body temperature $\left(38-39{ }^{\circ} \mathrm{C}\right)$. Upon verification of the loss of pain reflex, a midline abdominal incision was made. The small intestine was surgically exposed, $10 \mathrm{~cm}$ of jejunum ligated for perfusion and cannulated with plastic tubing (0.04 inch. i.d., 0.085 inch. o.d.). The ccannulated segment was rinsed with saline $\left(37^{\circ} \mathrm{C}\right)$ and attached to the perfusion assembly which consisted of a syringe pump (Palmer, UK) and a $60 \mathrm{ml}$ syringe connected to it.
The small intestine was gently handled during surgery in order to maintain an intact blood supply. The entire surgical area was then covered with saline-wet gauze $\left(38{ }^{\circ} \mathrm{C}\right)$ and was kept warm with a heating lamp. Blank perfusion buffer $(\mathrm{pH}$ 7.2) was first infused for $10 \mathrm{~min}$ followed by perfusion of test compounds at a flow rate of $0.2 \mathrm{ml} / \mathrm{min}$ for 90 min. Four concentrations of digoxin in phosphate buffered saline were perfused in the absence and presence of P-gp inhibitors erythromycin and clarithromycin in separate groups of animals. Outlet samples were collected every $10 \mathrm{~min}$ in microtubes. The volume of sample for each time interval was $2.0 \mathrm{ml}$. At the end of perfusion, the length of the segment was measured and the animal euthanitized by cardiac injection of saturated solution of $\mathrm{KCl}(345 \mathrm{~g} / \mathrm{L})$. Samples were stored at $-20{ }^{\circ} \mathrm{C}$ until analysis.

Preliminary experiments showed that no considerable adsorption of compounds on the tubing and syringe took place. Absorption or secretion of water was estimated by calculating the differences between inlet and outlet concentrations of phenol red (a nonabsorbable marker).

The study was reviewed and approved by the institutional ethical review board of Tabriz University of Medical Sciences, Iran (ref no. 8523) and adhered completely to the "Guide for the care and use of laboratory animals" [10].

\section{Data treatment and statistics}

Effective permeability coefficients $\left(P_{\text {eff }}\right)$ were calculated after correcting outlet concentrations for water flux on the basis of the ratio of phenol red concentrations of perfusion buffer entered and left for each sampling point (10 min). Permeability values were calculated using Eq 1.

$$
P_{\text {eff }}=-Q \ln \left[C_{\text {out }} / C_{\text {in }}\right] / 2 \pi \ldots \ldots . .
$$

where $Q$ is the flow rate $(0.2 \mathrm{ml} / \mathrm{min}), C_{\text {in }}$ and $C_{\text {out }}$ the respective inlet and outlet concentrations that had been adjusted for water transport during perfusion, $r$ is the 
radius of intestine (the most widely used estimate is $0.18 \mathrm{~cm}$ ) and $I$ is the length of the intestine measured after completion of perfusion.

The length of the intestine was measured in situ placing a piece of string along the intestine and measuring the string with a ruler. Permeability values are given as mean \pm SEM. Statistical difference between the permeability of digoxin in the presence and absence of macrolides was evaluated by twotailed Student's t-test. (SPSS, 13 version, SPSS Inc., Chicago, USA). Statistical significance was set at $p<0.05$.

To evaluate the quantitative functional role of $P$-gp, intestinal efflux inhibition ratio (EIR), ratio of $P_{\text {eff }}$ due to P-gp-mediated efflux transport $\left(P_{p-g p}\right)$ and passive permeability $\left(P_{P D}\right)$, were calculated. $P_{p-g p}$ was calculated by subtracting $P_{\text {eff, control }}$ from $P_{\text {eff,inh }}$ while $P_{P D}$ is equal to $P_{\text {eff,inh. Thus the EIR is calculated }}$ according to Eq 2.

$$
E I R=1-P_{\text {eff }, \text { control }} / P_{\text {eff }, \text { inh }}
$$

\section{RESULTS}

During single-pass perfusion studies, steady state was reached about $40 \mathrm{~min}$ after the beginning of perfusion and was confirmed by plotting the ratio of the outlet to inlet concentrations versus time. The results are plotted in Fig 1.

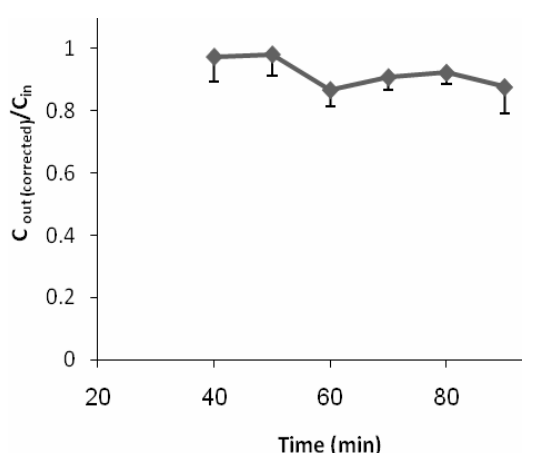

Fig 1: Plot of ratio of inlet and outlet concentrations $\left(\mathrm{C}_{\text {in }} / \mathrm{C}_{\text {out }}\right)$ vs time for digoxin (20 $\mu / \mathrm{ml}$ ) perfusion study. ( $n=4$, error bars represent $\mathrm{SD)}$
The mean effective permeability coefficient $(\mathrm{cm} / \mathrm{sec})$ of digoxin at concentrations of 5,10 , 15 and $20 \mu \mathrm{M}$ in perfusion solution were: 0.30 $\pm 0.01 \times 10^{-4}, 0.29 \pm 0.01 \times 10^{-4}, 0.32 \pm 0.03 \times$ $10^{-4}$ and $0.24 \pm 0.01 \times 10^{-4}$, respectively. In the presence of $150 \mu \mathrm{M}$ erythromycin as P-gp inhibitor in the perfusion buffer, at the above digixin concentrations, the permeability coefficient $(\mathrm{cm} / \mathrm{sec})$ values were: $0.49 \pm 0.02$ $\times 10^{-4}, 0.42 \pm 0.02 \times 10^{-4}, 0.48 \pm 0.02 \times 10^{-4}$ and $0.42 \pm 0.04 \times 10^{-4}$, respectively (Fig 2).

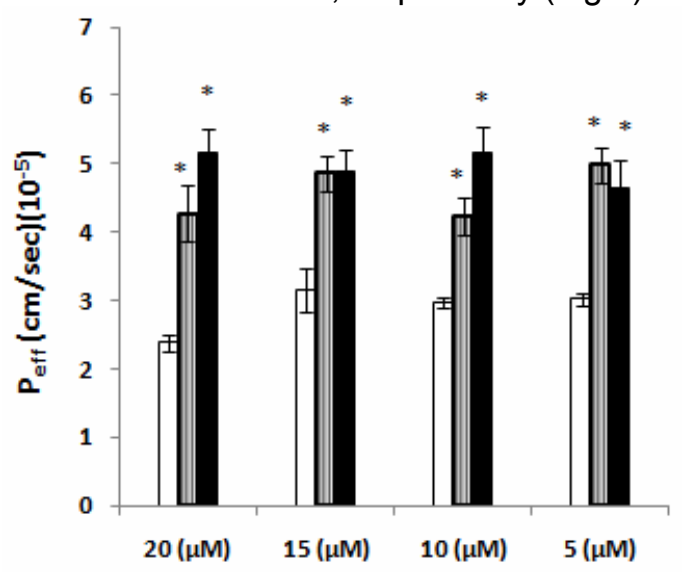

Fig 2: Intestinal permeability of digoxin in the absence and presence of P-gp inhibitors erythromycin and clarithromycin (150 $\mu \mathrm{M})$ :

control, $\square$ with erythromycin, - with clarithromycin; values are mean \pm SED

When the experiments were carried out in the presence of clarithromycin, another efflux pump inhibitor, the effective permeability values at the above concentrations of digoxin were $0.46 \pm 0.08 \times 10^{-4}, 0.51 \pm 0.07 \times 10^{-4}$, $0.48 \pm 0.06 \times 10^{-4}$ and $0.52 \pm 0.07 \times 10^{-4}$ $\mathrm{cm} / \mathrm{sec}$, respectively.

Two-tailed Student's t-test showed that the intestinal permeability of digoxin was significantly increased by erythromycin at all the four concentration of digoxin used ( $p<$ 0.05). Similar results were obtained with clarithromycin as a P-gp inhibitor. However, there was no significant difference between the $P_{\text {eff }}$ values of digoxin in different concentrations indicating no concentrationdependency for digoxin permeation. 


\section{DISCUSSION}

To evaluate the possible role of P-gp in absorption of digoxin, a jejunal segment of rat small intestine was used. In the intestine, P$\mathrm{gp}$ is expressed in the brush-border membrane of enterocytes and acts as a first line of absorption barrier by limiting influx. Secondly, P-gp facilitates the efflux of its substrates from the blood to the intestinal lumen [11]. On the other hand, intestinal metabolism is also an important determinant of bioavailability. Most of the liver drugmetabolizing enzymes are also present in the small intestine; however, the levels are generally much lower in the small intestine [12].

Digoxin is metabolized in rat with approximately $60 \%$ of plasma clearance being attributed to biotransformation by cytochrome P-450. Hepatic metabolism probably accounts for most of the biotransformation since in vitro incubation of digoxin with rat intestinal tissue showed that digoxin is not metabolized by rat enterocytes to any significant extent [12]. However, in another study with perfused rat small intestine, little metabolism in the small intestine was observed, and cytochrome P450 inducer treatment failed to change its metabolic profile [13]. These results suggest that the metabolism of digoxin is not dependent upon the cytochrome P-450 system in the intestine.

Therefore, in this study, the magnitude of possible P-gp-mediated drug interaction for digoxin and macrolides (erythromycin and clarithromycin) were compared at the absorption level. The single-pass intestinal perfusion model, with continuous perfusion of the lumen of an isolated segment of rat intestine with a solution of the drug, is particularly suitable for drugs with low absorption potential such as digoxin [2]. Since only a minor fraction of digoxin in the lumen is absorbed under control conditions, then an increase in the absorption rate is thus clearly evident from the corresponding decrease in the luminal level of digoxin relative to control experiments. The In the presence of erythromycin and clarithromycin, intestinal permeability of digoxin increased $1.40-1.80$ and. $1.54-2.18$-fold, respectively. Therefore, it is clear that at least a part of the observed clinical interaction between erythromycin and digoxin is due to increased absorption level of the latter. In almost all previous case reports, digoxin toxicity was attributed to altered gastrointestinal flora resulting in decreased metabolism of digoxin to digoxin-reduced products (DRPs) by gastrointestinal flora [6]. Moreover, some animal studies show the unexpected contrary results [14, 15]. For example, Budinha et al [13] investigated the influence of verapamil $(50 \mu \mathrm{M})$ as a P-gp inhibitor on digoxin transport using everted gut sac technique. In another study [15] using in situ circulated perfusion method, the effect of coadministration of another P-gp inhibitor, quinidine, on digoxin absorption clearance was examined. Both studies $[14,15]$ reported a significantly decreased absorption of digoxin in the presence of efflux pump inhibitors.

These results may be explained by the possible inhibitory roleeffect of verapamil and quinidine on organic anion transporter polypeptide (OATP) [5]. Digoxin is a substrate of OATP1B3 which was thought to be expressed only in the liver, but Glaeser et al [15] showed that it is also expressed in the intestine, and therefore, its inhibition results in reduced absorptive permeability of digoxin [16]. However, the results of the present study showed enhancement of digoxin intestinal absorption by inhibiting P-gp with macrolides which is in accordance with other previously reported studies [15,17]. Several pharmacokinetic studies in humans have indicated that P-gp inhibitors and inducers do not affect digoxin renal clearance [17]. Furthermore, increase in digoxin levels and toxicity have been observed only for orally co-administeration of digoxin and macrolides . Although it was suggested that erythromycin and clarithromycin had different potencies for 
inhibition of P-gp [18], our results showed no significant difference between the effects of the macrolides which confirm the results of the study conducted by Tsutsumi et al. [19]

Assuming complete P-gp inhibition by erythromycin, EIR for digoxin was found to be $0.30-0.44$, indicating that $30-44 \%$ of passive transport of digoxin is attenuated by P-gp-mediated transport. Based on permeability data for digoxin in the presence and absence of clarithromycin, the EIR value is $0.35-0.54$. According to previously published correlation between human and rat $P_{\text {eff }}$ values [9], the effective permeability coefficient for digoxin in human intestine is predicted to be more than $1.45 \times 10^{-4} \mathrm{~cm} / \mathrm{sec}$. On the other hand, the dose number (a criterion for solubility which is defined as the ratio of dose concentration to drug solubility) [20] of digoxin is 20.48; therefore, based on the previously published Biopharmaceutical Classification System (BCS) for drugs (which was derived from rat permeability and drug dose number) [21], digoxin can be classified as a BCS class IV drug, i.e., low permeabilitylow solubility drug (Do $>1, \mathrm{P}_{\text {eff (rat) }}>4.02 \times 10^{-5}$ $\mathrm{cm} / \mathrm{sec}$ ) (Fig 3). Therefore, as it can be seen in Fig 3 digoxin lies under the cutoff for highly permeable drugs which was set to be $5.09 \times 10^{-5} \mathrm{~cm} / \mathrm{sec}$ [21]. The intersections of dashed lines drawn at the cutoff points for permeability and dose/solubility ratio divide the plane in Fig. 3 into four explicitly defined drug categories $(I-I V)$.

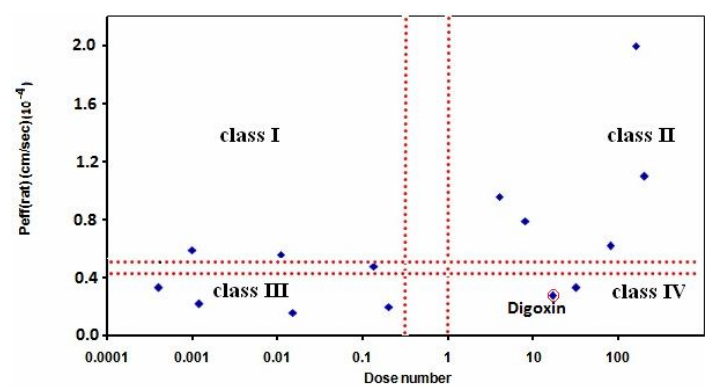

Fig 3: Classification of drugs based on dose number and rat $P_{\text {eff }}$ values which were previousely reported by authors $[21,22]$. Digoxin is added to this system as a class IV drug.
To confirm this finding, digoxin intrinsic dissolution rate (IDR) measurement is recommended which makes it possible to assess its category based on recently BCS [22]. Low and variable absorption for these drugs is anticipated because of the combined limitation of solubility and permeability. Formulation may improve the bioavailability of these drugs. However they are compromised by their poor intestinal membrane permeability. Strategies to improve both solubility and permeability should be worked out for these molecules, which may not be an easy task. However, obtaining this type of quality information will certainly improve drug design and help in optimizing candidates with "brick-like" properties. Consequently, the poor dissolution characteristics of digoxin dosage forms still remain an important bioavailability limiting step.

\section{CONCLUSION}

In this study, we found that erythromycin and clarithromycin enhanced the membrane permeability of digoxin in anaesthetized rats with no significant difference between their effects. Therefore, the most probable explanation for digoxin-macrolide interaction is inhibition of intestinal P-gp-mediated efflux of digoxin which leads to increased digoxin intestinal absorption. This finding should be considered when digoxin is prescribed together with other P-gp inhibitors and/or inducers.

\section{COMPETING INTEREST}

The authors report no conflict of interest.

\section{REFERENCES}

1. Pachot Jl, Botham RP, Haegele KD, Hwang $K$. Experimental estimation of the role of $P$ Glycoprotein in the pharmacokinetic behaviour of telithromycin, a novel ketolide, in comparison with roxithromycin and other macrolides using the Caco-2 cell model. J Pharm Pharm Sci. 2003; 6(1): 1-12.

2. Sababi M, Borga $O$, Hultkvist-Bengtsson $U$. The role of P-glycoprotein in limiting intestinal regional 
absorption of digoxin in rats. Eur J Pharm Sci. 2001; 14(1): 21-27.

3. Mayer U, Wagenaar E, Dorobek B, Beijnen JH, Borst $P$, Schinkel AH. Full blockade of intestinal $P$ glycoprotein and extensive inhibition of bloodbrain barrier P-glycoprotein by oral treatment of mice with PSC833. J Clin Invest. 1997; 100(10): 2430-2436.

4. Cavet ME, West M, Simmons NL. Transport and epithelial secretion of the cardiac glycoside, digoxin, by human intestinal epithelial (Caco-2) cells. $\mathrm{Br} J$ Pharmacol. 1996; 118(6): 13891396.

5. Yao HM, Chiou WL. The complexity of intestinal absorption and exsorption of digoxin in rats. Int J Pharm. 2006; 322(1-2): 79-86.

6. Bizjak ED, Mauro VF. Digoxin-macrolide drug interaction. Ann Pharmacother. 1997; 31(9): 1077-1079.

7. Seithel A, Eberl S, Singer K, Auge D, Heinkele G, Wolf $N B$, et al. The influence of macrolide antibiotics on the uptake of organic anions and drugs mediated by OATP1B1 and OATP1B3. Drug Metab Dispos. 2007; 35(5): 779-786.

8. Zakeri-Milani $P$, Valizadeh $H$, Islambulchilar $Z$, Damani S, Mehtari M. Investigation of the intestinal permeability of ciclosporin using the in situ technique in rats and the relevance of P-glycoprotein. Arzneimittelforschung. 2008; 58(4): 188-192.

9. Zakeri-Milani $P$, Valizadeh $H$, Tajerzadeh $H$, Azarmi $Y$, Islambolchilar $Z$, Barzegar $S$, et al. Predicting human intestinal permeability using single-pass intestinal perfusion in rat. $J$ Pharm Pharm Sci. 2007; 10(3): 368-379.

10. Ernest $D$, Alfert $E$, Brenda $M$, Cross $B M$, McWilliam $A A E$. CCAC, Guide to the care and use of experimental animals, Canadian Council on Animal Care. 1993.

11. Anwar-Mohamed A, O.S. El-Kadi A. P-glycoprotein effects on drugs pharmacokinetics and drugdrug-interactions and their clinical implications. LJPCP. 2012; 1(51150): 1-15.

12. Liu L, Mak E, Tirona RG, Tan E, Novikoff PM, Wang $P$, et al. Vascular binding, blood flow, transporter, and enzyme interactions on the processing of digoxin in rat liver. J Pharmacol Exp Ther. 2005; 315(1): 433-448.
13. Liu S, Tam D, Chen X, Pang KS. P-glycoprotein and an unstirred water layer barring digoxin absorption in the vascularly perfused rat small intestine preparation: induction studies with pregnenolone-16alpha-carbonitrile. Drug Metab Dispos. 2006; 34(9): 1468-1479.

14. Budihna MV, Strojan P. Ca2+ channel antagonists inhibit the intestinal absorption of digoxin in the guinea-pig. Eur J Pharmacol. 1993; 230(3): 301-305.

15. Su SF, Huang JD. Inhibition of the intestinal digoxin absorption and exsorption by quinidine. Drug Metab Dispos. 1996; 24(2): 142-147.

16. Glaeser H, Bailey DG, Dresser GK, Gregor JC, Schwarz UI, McGrath JS, et al. Intestinal drug transporter expression and the impact of grapefruit juice in humans. Clin Pharmacol Ther. 2007; 81(3): 362-370.

17. Greiner B, Eichelbaum M, Fritz $P$, Kreichgauer HP, von Richter $O$, Zundler J, et al. The role of intestinal P-glycoprotein in the interaction of digoxin and rifampin. J Clin Invest. 1999; 104(2): 147-153.

18. Wang L, Kitaichi K, Hui CS, Takagi K, Sakai M, Yokogawa $K$, et al. Reversal of anticancer drug resistance by macrolide antibiotics in vitro and in vivo. Clin Exp Pharmacol Physiol. 2000; 27(8): 587-593.

19. Tsutsumi $K$, Kotegawa $T$, Kuranari M, Otani $Y$, Morimoto T, Matsuki S, et al. The effect of erythromycin and clarithromycin on the pharmacokinetics of intravenous digoxin in healthy volunteers. J Clin Pharmacol. 2002; 42(10): 1159-1164.

20. Rinaki E, Valsami G, Macheras P. Quantitative biopharmaceutics classification system: the central role of dose/solubility ratio. Pharm Res. 2003; 20(12): 1917-1925.

21. Zakeri-Milani $P$, Valizadeh $H$, Tajerzadeh $H$, Islambulchilar Z. The utility of rat jejunal permeability for biopharmaceutics classification system. Drug Dev Ind Pharm. 2009; 35(12): 1496-1502.

22. Zakeri-Milani $P$, Barzegar-Jalali $M$, Azimi $M$, Valizadeh $H$. Biopharmaceutical classification of drugs using intrinsic dissolution rate (IDR) and rat intestinal permeability. Eur $J$ Pharm Biopharm. 2009; 73(1): 102-106. 